\title{
Tres documentos y situaciones de la masonería en Melilla y Tetuán
}

\author{
José A. Ferrer Benimeli
}

\section{Muerte en Melilla}

El 30 de diciembre de 1893, en el número 71 de la Gaceta Oficial del Grande Oriente Ibérico, aparecía un curioso clogio fúncbre con cl título de "Datos biográficos y mucrte en Melilla del Ilustre Hermano José Valcro, grado 30", que por su interés merece la pena sea recuperado a la memoria histórica por cuanto tiene de crítica y análisis de la política africanista de finales del siglo XIX. Decía así:

Sirvió a Valero de cuna la ciudad de Valencia, que hoy premia al ilustre fancmasón y al infatigable africanista, dando su nombre a una de sus calles.

Activo y emprendedor como los hijos de aquella hermosa ciudad, a pesar de tener en su seno todos los elementos de riqueza y progreso permanece estacionaria, no acomodándose con el quietismo de la población en que vio la luz. demostró desde muy niño las grandes iniciativas que en aquel rostro varonilmente atractivo, en que vagaba sin cesar una sonrisa tan grata como la hermosa huerta valenciana, se reflejaban unidas a las generosas ideas del patriota y del colonizador.

Dotado de una rara inteligencia, terminó en breves años su carrera, y casi un niño, ocupó una plaza de catedrático en el Colegio de Almansa.

La guerra civil asolaba nuestra noble tierra, y Valero que entendía que las ideas de civilización y de progreso deben defenderse en todos los terrenos, y que los ideales de la paz deben dejarse para cuando la guerra termina, cambió la toga del catedrático para vestir el honroso uniforme del soldado.

El Cuerpo Administrativo del Ejército se vanagloría hoy a justo título, de haber tenido en su seno al comisario de guerra $D$. José Valero, quien mientras hubo en la Península enemigos de la causa de la libertad, al par que cumplia con los deberes de su cargo, se batía constantemente como soldado en los puntos de mayor peligro, y que terminada aqui la guerra pasó a Cuba, donde su actividad, su valor, su inteligencia y su honradez, llevándole a cumplir en más de una circunstancia delicadisimas misiones, le valieron la confianza y particular estimación de todos sus jefes, y en especial del general Martínez Campos, pues gracias a su arrojo y serenidad, solo, y perdida la escolta que le acompañaba, salvó una crecida conducción de caudales, y con ella la difícil situación de una parte del ejército que sin él se hubiera visto en la imposibilidad de resistir.

Valero se batía siempre que había combate, y cuando la jornada terminaba se dedicaba a las cuentas y a la previsión de los mil detalles del Cuerpo especial a 
que pertenecia, y en el campo, y en la ciudad, y en las Logias, en donde las habia y a las que nunca faltaba, se mostraba siempre con aquella dulce sonrisa de su inmaculada conciencia y con los rasgos característicos de su ferviente patriotismo, jamás reñidos con lo jovial y cariñoso de su trato.

Terminada la guerra de Cuba, vuelve Valero a la Península, y entendiendo que en la paz deben aprovechase todas las inteligencias para el mejor desarrollo de los intereses sociales, se dedica al estudio de aquellos problemas que pueden tender al engrandecimiento de su patria.

La travesía del Allántico, le habia hecho pensar en los problemas colonizadores, y su breve visita al Africa, le hizo comprender que ella sería el teatro de las operaciones de los ejércitos europeos en el siglo XX.

Nosotros le hemos oido quejarse con dolor de esos figurones de relumbrón que a si propios se llaman hombres de Estado, porque sin méritos para ello han llegado en los diferentes partidos políticos a escalar determinados puestos, sin haberse preocupado jamás de los peligros que para nuestra independencia tendría el establecimiento de una potencia cualquiera entre el Estrecho de Gibraltar y la cordillera del gran Atlas.

España no sabe acomodarse a esos olvidos, porque España es un pueblo viril de grandes aptitudes, que no puede resignarse ni aceptar las ideas de cierto filósofo que con pretensiones de consejero absoluto, ha dicho que nosotros debemos prescindir de pasiones coloniales, dedicando nuestros esfuerzos a reconstruir la Hacienda, a lo que Valero respondio:

"Sin comercio exterior no hay riqueza, y sin una buena política internacional no hay comercio exterior. Y si el resultado para la prosperidad pública no es muy satisfactorio, con esas absurdas ideas políticas y económicas, tampoco lo es, teniendo en cuenta nuestra futura independencia. ¿Es que la filosofía no enseña nada de estrategia, y no revela lo que pudiera ser de España si Marruecos fuese una posesión francesa o inglesa? Pues el que no sabe de una cosa que no hable de ella, y el que sólo sirva para estudios especulativos, que no tome parte en la vida pública, que es grande error desconocer el contraste absoluto entre un pueblo y un ejército valiente hasta la heroicidad, y de una política exterior, tímida hasta la cobardia".

Fiel a estas ideas, Valero adivinó en la campaña y la colonización de Joló la incógnita de un gran pensamiento, y proclamando que lo que aquí hacen falta son hombres de corazón y de patriotismo que se pongan al frente del movimiento colonial, partió para el Golfo de Guinea, donde realizó valiosisimas exploraciones, llegando a donde nadie había llegado, dejando constituidas factorias, y trayendo multitud de proyectos colonizadores que le hicieron sufrir un verdadero calvario al acudir a los hombres de la política y de la Banca en demanda de ayuda para realizar un bien en pro de la humanidad y de la patria.

No desiste por esto en su campaña africanista. Pide un destino para Ceuta, creyendo encontrar alli algo que sirva a sus estudios; sus especiales aptitudes hacen que el Gobierno le traiga a desempeñar una cátedra en la Academia de su Cuerpo en Avila, y alli le sorprenden los sucesos de Melilla que, despertando su constante idea de que la posesión de Marruecos es para España cuestión de vida o 
muerte, le hacen marchar allá, y en un día en que faltando viveres en el fuerte de Cabrerizas era preciso enviar a todo trance un convoy, se presta voluntario a conducirlo, y en medio de un fuego que diezma las fuerzas, llega tras ruda lucha hasta la puerta del fuerte, y al decir con la constante sonrisa de su indomable valor: "Caballeros, cómo tiran", una bala le atraviesa el estómago y cae al suelo al llegar al logro de su empeño.

iQue la generosa sangre de Valero sea símbolo de redención y que España llegue a ocupar el puesto que de derecho le corresponde! La patria ha perdido uno de sus más entusiastas adeptos, y como dice el ilustrado capitán de Ingenieros $D$. Eusebio Jiménez, sobre la fosa que guarda sus preciados restos, debla ponerse una lápida con esta inscripción:

Aquí yace un buen español.

Vivio para su patria y murió por ella en el campo de batalla.'

\section{El Alto Comisario de Marruecos y los Masones}

Uno de los momentos de máximo esplendor de la masonería española en el siglo $\mathrm{XX}$ fue sin duda alguna la $2^{\mathbf{4}}$ República, y en especial durante el primer bienio en el que un buen número de cualificados masones ocuparon puestos claves en el Gobiemo y en la Cámara Constituyente. Basta recordar que en el primer gobierno provisional de la República eran masones, al menos seis ministros, Alejandro Lerroux (Ministro de Estado), Alvaro de Albornoz (Ministro de Fomento), Diego Martínez Barrio (Ministro de Comunicaciones), Fernando de los Ríos (Ministro de Justicia), Marcelino Domingo (Ministro de Instrucción Pública) y Santiago Casares Quiroga (Ministro de Marina); y que unos meses depués lo sería también Manuel Azaña (Ministro de la Guerra).

Algunos de ellos detentaban tambićn altos cargos dentro del Grande Oriente Español, pues en la Asamblea Nacional celcbrada los días 5 y 6 de julio de 1931 en que fue elegida la Comisión Permanente del G.O.E. fueron designados como Presidente, Diego Martínez Barrio (Ministro de Comunicaciones); Primer Vice-Presidente, Marcelino Domingo (Ministro de Instrucción Pública); $2^{\circ}$ Vice-Presidente, Rodolfo Llopis (Director General de 1" Enseñanza); Gran Orador, Emilio Palomo (Gobernador de Madrid); Vocal $1^{2}$, Fernando de los Ríos (Ministro de Justicia); Vocal $3^{2}$, Pedro Rico (Alcalde de Madrid); Vocal 5, Demófilo de Buen (Consejero de Estado), y otros muchos entre los que figuraban siete diputados de los más de ciento cuarenta diputados a Cortes que por aquel entonces pertenecían a la masonería. ${ }^{2}$

(1) Gaceta Oficial del Grande Oriente Ibérico, año VII, Madrid 30 de diciembre de 1893, $\mathrm{n}^{2} 71$, pág. 629. Esta nota biográfica a su vez la toman del Boletín Oficial del Grande Oriente Nacional de Espafta, precedida del siguiente comentario: "Valero ha muerto gloriosamente en el Rif a donde fue voluntariamente a luchar por su patria. Que reciba en la historia y en la etemidad la recompensa de sus méritos y bondades".

(2) Cfr. FERRER BENIMELI, José A., Masonerla española coniemporánea, Madrid Siglo XXI de España Ed., 1980, vol. II, págs. 67-108; 215-224; FERRER BENIMELI, José A., La Masonerla y la Constitución de 1931, Rev. Cuademo de Investigación Hitórica [Madrid], n 5 (1981) 217-274. Sobre el por qué de estos nombramientos y su relativo valor masónico, cfr. CRUZ OROZCO José Ignacio, Actuación polúica de la masonería en la Segunda República. Algunas precisiones [en prensa]. 
Pues bien, a pesar de esta situación aparente de privilegio en que parece ser se encontraba en aquel momento la masonería española, sin embargo en el protectorado español de Marruecos la realidad era muy distinta según se desprende de la documentación de la época. Así, en una carta fechada en Tetuán el 15 de diciembre de 1931, dirigida desde la logia Oriente $n^{2} 451$ del Grande Oriente Espaniol, y que decía lo siguiente:

"Hace años nos reunimos en estas tierras un grupo de Hermanos, pequeños por el número, pero grandes por nuestros entusiasmos sin límites. Se encontraba en esta ciudad africana el funesto Directorio en pleno. Orgullosos y soberbios como buenos tiranos, no permitian a nadie expresarse sus ideas. Una nube de confidentes delataban hasta los menores gestos de los amantes de la Libertad y los Derechos del Hombre.

Unos cuantos Hermanos, los siete indispensables, levantamos columnas arrastrando gallardamente la responsabilidad y el peligro.

Calladamente, silenciosamente, fuimos labrando estas tierras vírgenes con el arado de la cultura y la razón, para sembrar la semilla de las ideas de todos, el amor tolerante de la ley justa y a los derechos ciudadanos. iSi viérais, Venerable Hermano y queridos hermanos, qué hermosos frutos hemos recogido y estamos recogiendo! La cosecha ha sido superior a nuestros cálculos. Nuestros ideales grabados han quedado en nuestras almas forjados en tremendos golpes de adversidad dados sobre el yunque de la tirania en ininterrumpida persecución odiosamente reaccionaria.

Nuestro Venerable y otros queridos hermanos fueron encarcelados no recobrando la libertad hasta que la Patria despierta, al fin, sacudio el linaje borbónico que la esclavizó durante siglos...

Hoy contamos con logias hermanas en todas las poblaciones africanas y plazas de soberania.

Al constituirnos con nuestro nombre de Oriente $n^{2} 451$ de la obediencia del Grande Oriente Español cumplimos gustosisimos el sagrado deber fraternal de enviaros nuestro sincero y cariñoso saludo ofreciéndoos al mismo tiempo nuestro modestisimo pero incondicional apoyo.

Os rogamos Venerable Maestro y queridos hermanos volváis la vista algunos momentos hacia estas tierras africanas donde tantos hermanos han dado su vida y su sangre por la paz y la civilización y no olvidéis un momento que en plena República española tenemos un Alto Comisario, Don Luciano López Ferrer, antiguo diputado ciervista, completamente reaccionario, para quien no ha llegado aún la hora de la verdadera Libertad cuya base y fundamento está en el respeto absoluto a todas las ideas.

Mantener a este hombre aqul es una burla sagrienta al pueblo español y a la República española.

;Gracias a que la segunda autoridad del Excmo. Sr. D. Emilio Zapico. Delegado General, es hermano nuestro y hombre republicano y demócrata por excelencia, miembro activo y entusiasta de la francmasonería!

En espera de vuestras gratas noticias, recibid Venerable Maestre y queridos hermanos el triple abrazo fraternal y ósculo de paz que por este conducto os en- 
vian todos los obreros de esta respetable Logia". ${ }^{3}$

Sin embargo el Delegado General poco pudo hacer, pues al poco ticmpo era destituido por el Alto Comisario, de la misma forma que lo scrían Miguel Cabancllas, Gcneral en Jefe de las Fuerzas Militares; Cristóbal de Lora, Jefe Superior de Policía de Marruecos; y Sánchez Plaza, Comandante de Caballería y Jefe de la Mehal-la de Tetuán, como se recoge en la carta que un par de meses más tarde, el 8 de febrero de 1932, enviaba a Madrid la logia Trafalgar $n^{2} 20$ de Algeciras, haciendo una angustiosa llamada de atención y pidiendo la destitución del Alto Comisario:

"Llega hasta este Taller la noticia por conducta que nos merece entero crédito de que el Alto Comisario de Marruecos, Sr. López Ferrer, monárquico recalcitrante de toda su vida, hombre de espítitu reaccionario, viene realizando una campaña persecutoria contra todos los masones del protectorado Marroqui, hasta el punto de que los va eliminando de los cargos oficiales con que fueron nombrados al advenimiento de la República.

Como no podemos permanecer cruzados de brazos ante campaña tan inicua $e$ insidiosa esta Respetable Logia Trafalgar eleva su más enérgica protesta contra la incalificable conducta del Sr. Ferrer y ruega a V. querido hermano tome buena nota de ésta y haga cuantas gestiones sean precisas para que dicho Comisario sea destituido de un cargo que nunca debió ocupar. Debemos advertir que el ya tristemente Sr. López Ferrer se jacta de las destituciones de nuestros muy queridos hermanos Cabanellas, Zapico, Lora y Sánchez Plaza.

Recibid con este motivo el testimonio de nuestro fraternal abrazo".4

Poco después la logia Luis Simarro $n^{2} 3$, de Madrid, con fecha 24 de febrero de 1932, respondía a la carta remitida por la logia Trafalgar $n^{2} 20$ de Algeciras, en estos términos:

"Mi taller me encarga comunique a ese Respetable que a su debido tiempo se recibió vuestra fraternal plancha del 8 del actual, en la cual nos dábais cuenta de la persecución de que son objeto nuestros hermanos de Marruecos por el Alto Comisario.

Su lectura, dada en tenida del 19 causó honda emoción entre los hermanos asistentes, los cuales hacen votos porque aquella termine.

Ya tentamos noticias, con anterioridad de este desagradable asunto, noticias que habian llegado a oidos de los consejeros del Oriente y de hombres [hermanos] que ocupan altos puestos en la política, los cuales esperan ocasión a propósito para que se haga sentir su influencia en favor de dichos hermanos.

Con motivo de la última asamblea han llegado a estos Valles hermanos de allende el estrecho y han expuesto su situación en lugar conveniente; y os puedo asegurar que muy pronto la situación de la Masonería en Africa habrá cambiado.

Por de pronto, dichos hermanos vuelven a sus Valles satisfechos y confiados en que la Justicia alumbrará en Marruecos".

(3) Archivo Historico Nacional. Salamanca [A.H.N.S.], Fondo Masoneria, Legajo 359-A, Exp. 26.

(4) Ibidem.

(5) Ibidem. 
El optimismo que se desprende de esta carta, que va firmada por Florencio Alvarez, ${ }^{6}$ no parece ser correspondió a las espectativas puestas en una rápida solución del problema, pues en el libro de Actas de la Gran Logia Regional de Marruecos, en sesión celebrada en agosto de 1932, se recoge un testimonio del Venerable hermano Arlandis que es bastante elocuente y que proporciona una serie de datos de indudable interés para reconstruir la historia y el porqué de la persecución masónica emprendida por el Alto Comisario que con total impunidad destituyó de sus puestos de mando tanto civiles como militares a todos los masones que ocupaban puestos calve en Marruecos, y en unos momentos en los que - paradógicamente - en el Gobierno Central de Madrid y en el Congreso de los Diputados los masones copaban toda una serie de cargos de gran importancia, lo que confirma la tesis de José Ignacio Cruz Orozco sobre el escaso valor $\mathrm{o}$ influjo que tuvieron los nombramientos masónicos de algunas personalidades políticas del momento. ${ }^{\text {? }}$

El libro de Actas en cuestión se expresa así:

"Concedida la palabra en bien general de la Orden hace uso de la misma el V.H. Arlandis, quien dice es vergonzoso lo que está ocurriendo con la Francmasonería en Marruecos, en donde son perseguidos todos los $\mathrm{HH}$. en forma despiadada, lesionándolos en sus intereses y en algo más grave cual es la propia estimación, puesto que caprichosamente y sin causa que lo justifique se encuentran los funcionarios militares y civiles trasladados a España y otros a sitios que no les corresponde. Dice que es hora de que se haga lo posible porque termine esta persecución. Se duele de que no obstante las quejas dadas el G.C.F.S. no se haya conseguido nada lo que ha alentado a las autoridades profanas en la campaña descarada que han emprendido contra la orden, censurando que se consienta este estado de cosas por las altas autoridades masónicas.

El Gran Secretario H. Lora, pide la palabra para una cuestión de orden. Hace historia de todo. Recuerda que el Alto Comisario D. Luciano López Ferrer en las primeras declaraciones que hizo en un banquete fueron que pensaba terminar con los "masoncillos de Marruecos" sin saber que lo estaba escuchando más de un masón entre otros el H. Miguel Cabanellas General en Jefe de las Tropas militares. Varios $H H$. asienten a lo dicho por el $H$. Lora.

Continúa diciendo que uno de los días que despachaba asuntos oficiales con el Alto Comisario, ignorando este que el que habla es masón, le preguntó sin conocía al "rey Baltasar", y al decirle que no, le dijo que el rey Baltasar era Cabanellas a lo que el $H$. Lora contestó que él "creía que Cabanellas era el General de las fuerzas republicanas en Marruecos" respondiéndole el Alto Comisario, que no, que Cabanellas tenía antes toda su confianza y por eso se alegró que viniera a Marruecos, pero que dejó de tenerla cuando se enteró que era masón.

A los pocos días, presentó el $H$. Lora, una discusión del Alto Comisario con el

\footnotetext{
(6) El domicilio social de la logia Luis Simarro $n^{e} 3$ era, calle Francisco Ferrer (antes Príncipe) $n^{e} 12-2^{2}$. Madrid.

(7) Cfr. nota 2.
} 
Delegado General V.H. Emilio Zapico, el que salió en defensa del H. Oros que fue injustamente castigado. $Y$ al decirle el Alto Comisario que lo hacia porque sabia que era masón, y asegurar que le merecían muy mal concepto todos, profirió palabras injuriosas para la orden no teniendo más remedio el $\mathrm{H}$. Emilio Zapico que rogarle no siguiera por ese camino porque lo estaba ofendiendo puesto que él también pertenecía a la orden.

El Alto Comisario dio orden al $\mathrm{H}$. Critóbal de Lora, que no volviera a despachar para nada con el General Jefe Miguel Cabanellas y con el Delegado General Emilio Zapico, cuya orden dio también a todos los Jefes de Servicios del Proteciorado y que al darle esa orden le dijo el Alto Comisario que no quería nada con los masones. Que él había pedido al Gobierno destinaran a la zona a Zapico el que se encontraba de Cónsul General en Magico, pero que sabiéndole masón no le quería tener un momento más a su lado. Ordenó al H. Lora, entonces Jefe de la Policía del Protectorado, que vigilara a Cabanellas y Zapico y le diera cuenta de todos sus pasos. Así lo prometió el H. Lora y se retiró.

A los pocos dias, volvió el Alto Comisario D. Luciano López Ferrer a sacar la conversación de los masones, injuriándolos $e$ insultándolos en forma tan soez $e$ inadecuada que el $\mathrm{H}$. Cristóbal de Lora no tuvo más remedio que rogarle retirara lo dicho por estarle ofendiendo en lo más intimo de sus ideales.

Desde este momento rompió $D$. Luciano López Ferrer toda relación con el $\mathrm{H}$. Lora, tanto oficial como particularmente.

Dice que el Alto Comisario se indignó al darse cuenta que la orden masónica tiene en Marruecos una fuerza insospechada.

Encargó por todos los procedimientos una lista de HH.; lista no completa que llevó su Ayudante Comandante de Infantería D. Antonio Villalba al Director de Marruecos y Colonias D. Antonio Canovas, para de común acuerdo con el Alto Comisario emprender la campaña contra los masones, cuya campaña dio inmediato resultado siendo destituido en primer lugar:

El H. Miguel Cabanellas General en Jefe de las Fuerzas Militares.

El H. Emilio Zapico, Delegado General de la Alta Comisaría que fue obligado a pedir traslado a Puerto Rico en donde se encuentra de Cónsul General.

El H. Cristóbal de Lora. Jefe Superior de Policía de Marruecos que estuvo detenido 6 días en Melilla sin causa justificada.

El H. Sánchez Plaza, Comandante de Caballería y Jefe de la Mehal-la de Tetuán que quedó disponible.

El H. Hassan Ben Mohamed Hehe que fue destituido de su cargo a las b́rdenes del Jefe Superior de Policía.

El H. Carlos Daiz de Tejada, Jefe local de Policía de Larache.

El H. Ismael Mendoza, Jefe local de Alcazarquivir.

El H. Roberto Marauri, Inspecto de Aduanas.

El H. José Alberola, Presidente de la Junta Municipal.

El H. Hach Aldeselan Ben Muna, Vicepresidente de la misma.

El H. José Gomir, Intérprete de la Delegación General.

El H. Rogelio González, Intérprete de la Delegación General.

El H. Tomás Orós Giménez, oficial de correos de Alcazarquivir. 
El H. Balboa de la Junta Municipal de Alcazarquivir.

El H. Alejandro García Menéndez Teniente de las Intervenciones Militares.

Otros HH. más que no recuerda en estos momentos.

Dice que nuestra defensa se inició mal desde el primer momento. Que el $\mathrm{H}$. José Navarro Díez, con la mejor intención suscribió un documento que repartió entre todos los diputados pertenecientes a la orden en el que se citaban pequeños discos (?) locales, sin apuntar directamente a la cabeza con lo que a juicio del $\mathrm{H}$. Lora no se consiguió más que un desgaste inútil de energía.

Asegura que le han encargado un informe serio, sensato, ajustado a la verdad, con casos concretos, avalado por esta Gran Logia y dirigido al G.C.F.S. para que por dicho Alto Organismo se haga de él el uso más conveniente para la mejor defensa de la orden.

Expresa la seguridad de que pronto cambie esta situacion".

El cambio, sin embargo, no llegó hasta el 20 de enero de 1933, cuando el hasta entonces Gobernador de Barcelona y futuro ministro de la Gobernación en 1936, Juan Moles, sustituyó como Alto Comisario de Marruecos ${ }^{9}$ al monárquico Luciano López Ferrer quien, paradógicamente, ejerció su cargo desde el 6 de junio de 1931 es decir durante el llamado bienio reformista de la República cuyos intereses no gozaron precisamente de su simpatía a juzgar por la actuación que tuvo al destituir y relegar poco a poco a todas aquellas personas que, al advenimiento de la República, habian sido nombradas y colocadas en puestos de responsabilidad.

Destituciones -especialmente de masones- que tuvieron lugar a pesar de los presuntos intereses masónicos de los dirigentes de la $2^{\mathbf{t}}$ República, quienes no parece ser ignoraran lo que sucedía en Marruecos, no sólo a través de la correspondencia masónica citada, sino también por la visita que el Ministro de Instrucción Pública, Fernando de los Ríos, -y destacado masón-, realizó a Marruecos en diciembre de 1931, y la que en junio de 1933 - pocos meses después de la destitución de López Ferrerhizo el entonces Ministro de la Gobernación, Casares Quiroga, también destacado masón.

El porqué de esta situación y su justificación jurídica hay que buscarla precisamente en el decreto del 3 de junio de 1931 por el que se reorganizaban las Fuerzas Militares de Marruecos, dentro del espítitu de reformas emprendidas por Azaña desde su ministerio de la Guerra, ${ }^{10}$ dando primacía al Alto Comisario que quedó situado por encima del Jefe Superior de las Fuerzas Militares. Esto hizo que las fricciones entre el Alto Comisario, Luciano López Ferrer ${ }^{11}$ y el Jefe Superior del Ejército del Protectorado, general Cabanellas —ambos nombrados el 6 de junio de 1931 - fueran frecuentes. ${ }^{12}$

(8) A.H.N.S., Leg. 499-A, Exp. 27: Libro de Actas de la Gran Logia Regional de Marnecos.

(9) MORALES LEZCANO, Victor, Espafia y el Norte de Africa: El Protectorado en Marruecas (1912-56), Madrid, UNED, 1984, pág. 217.

(10) Azafia ingresaría también en la Masonería el 2 de marzo de 1931 pero tampoco parece ser que su actitud de masón influyera demasiado para evitar las destituciones de sus "hermanos" de Marruecos.

(11) Luciano López Ferrer sustituyó en el cargo al general Sanjurjo nombrado apenas unas semanas antes, el 23 de abril de 1931.

(12) Véase, por ejemplo, el artículo de Cabanellas "La situación de Marruecos", publicado en El Sol el 13 de noviembre 1931, que fue replicado por el mismo López Ferrer en la edición del día siguiente. 
Otro de los temas que se planteó la masonería española de Marruecos durante la 2: República fue el del nacionalismo. Movimiento que queda bastante bien reflejado en una plancha o discurso masónico que lleva la firma de $\operatorname{Hassan}^{13}$ y que tras la invocación ritual de "A la Gloria del Gran Arquitecto del Universo" decía lo siguiente:

"Queridos Hermanos: En este acto trascendental, en el que nos visita el Muy Respetable Gran Maestre de la Orden, muy Ilustre hermano Diego Martinez Barrio, no quiero desperdiciar la ocasión de trazar unas lineas para que este distinguido hombre público sepa de los labios de un hermano que ha jurado decir la verdad en todo momento y ocasión, cual es la realidad de la situación del país protegido por España, cuna de nuestros antepasados".

NACIONALISMO MUSULMAN. A este enunciado se le viene dando un cariz y una importancia de la que carece absolutamente. A mi se me tilda de jefe de este movimiento imaginario. El nacionalismo, en la Zona de Protectorado, es, en realidad, el deseo de muchos musulmanes de instruirnos en lo moderno, sin abandonar lo que es peculiar nuestro. Comprendemos que, con relación a Europa, estamos atrasados de un siglo, y es nuestro más ferviente deseo el de ver si esta laguna podemos salvarla en el menor plazo de tiempo posible. Como la misión que aqui se ha impuesto España no es otra, venimos a sacar en consecuencia que el primer nacionalista de la Zona es el propio pais protector.

Muchas veces he dicho a mis queridos hermanos que la direccion y administración de nuestra Zona, al no estar en manos de España estaría en las de cualquiera otra potencia europea, con la agravante de que con ella no habríamos de tener los lazos de amistad y parentesco que con los españoles nos unen. Los protectores de la Zona vecina están bien impuestos de la afinidad entre españoles y musulmanes, y saben, además, que todos los favores que recibimos de España son comunicados a nuestros compatriotas de la Zona francesa, quienes envidian nuestra suerte en lo que se refiere en la parte moral y política, aunque en lo económico ellos nos aventajen.

Tal vez por esto los franceses, empleando su tradicional diplomacia, tratan de prevenir contra los pretendidos nacionalistas, y muy especialmente contra mí, a los gobernantes de Madrid y de Tetuán, creando un fantasma alli donde sólo existe un legítimo deseo de superación. Y lo más triste del caso es que los gobernantes, prestándose al juego francés, nos tratan como si en efecto fuéramos conspiradores y gente peligrosa. Ast encubren los franceses su notorio imperialismo en la otra Zona de Marruecos.

Sin temor a equivocarme, y conociendo profundamente a mis correligionarios, puedo afirmar que no hay en toda la Zona un musulmán que levante el brazo contra España, y menos aún contra la España republicana que nos ha traido la libertad de conciencia.

(13) Posiblemente Abder-elam B. Laarbi Buma, según consta en los papeles del A.H.N.S., Fondo Masonería, Leg. 351-A, Exp. 21. 
Las Logias están llamadas a realizar en Marruecos un trabajo de compenetración entre protectores y protegidos. El masón está obligado a informar a sus hermanos españoles de cuanto malo pueda fraguarse contra el pals protector. y del mismo modo. las Logias del Protectorado deben velar por que se cumpla fielmente, con equidad y justicia, cuanto se relaciona con los protegidos, tomando su defensa cada vez que la ocasión lo requiera.

Haciendo un poco de historia, en la guerra de la independencia de la América española llegó un día en que se declaró la lucha a muerte y ésta duró, encarnizada y cruel, hasta el encuentro del General Morillo con Bolivar en la ciudad de Trujillo (Venezuela). Alli se conocieron como hermanos masones, se dieron el abrazo fraternal y terminó la guerra a muerte, comenzando a trazarse el Tratado de Paz, entre madre e hijas. ${ }^{14}$

PANISLAMISMO. En el último Congreso celebrado en Palestina hace pocos meses, quedó también desvanecido el fantasma de la guerra santa con la declaración pública de que todo musulmán no tiene más obligación patriótica que la de actuar en la tierra donde ha nacido, y que sólo tres ciudades en el mundo pertenecen al islamismo universal: La Meca, Medina y Jerusalem. las que deben ser consideradas como patrimonio de la religión mahometana, y, en consecuencia, todo musulmán debe contribuir a su engradecimiento.

RESUMEN. Aqul no existe nacionalismo de ningún género, sino un deseo de ilustrarnos y entrar francamente en la ruta del progreso y de la civilización. A esto debe España ayudarnos francamente, sin entretenerse en buscar topicos falsos ni en crear fantasmas que se desvanecen al primer soplo de la realidad. Este del nacionalismo no es más que un juego de los franceses para encubrir su imperialismo y desviar la atención hacia otros asuntos. He dicho. ${ }^{15}$

La postura oficial que, ante este asunto, adoptó la Gran Logia de Marruecos, de la federación del Gran Oriente Español, está recogida en un Decreto fechado en Tetuán el 12 de julio de 1933, y que decía lo siguiente:

Rogelio González Belloto, Grado 13 del Rito Escocés Antiguo y aceptado, Diputado Gran Maestre accidental de la Gran Logia Regional de Marruecos. A cuantos el presente vieren.

SABED:

Que en Tenidas celebradas por esta Gran Logia, en los días cuatro y cinco del mes en curso, se sometió a laborioso y amplio debate la cuestión "Nacionalismos" como idea politica y palpitante en la actualidad y en pugna siempre con nuestros más esenciales postulados.

Que en virtud de dichos principios básicos y fundamentales esta Gran Logia Regional considera incompatible el ideal masónico con los nacionalismos, cualquiera que sea el matiz político de éstos; por lo cual y en uso de las facultades conferidas en el párrafo $1^{9}$ del art. 63 de los Estatutos y Reglamentos Generales.

(14) Acerca de esta visión un tanto simplista del problema Cfr. FERRER BENIMELI, Jose A., Bolivar y la Masonerla, Revista de Indias [Madrid], vol. XLIII, $\mathrm{n}^{2} 172$ (julio-diciembre 1983) 631-687.

(15) A.H.N.S., Fondo Masonerí, Leag. 351-A, Exp. 21: Nacionalismo musulmán. 
DECRETO:

Artículo $1^{\circ}$.- La Gran Logia Regional de Marruecos se declara incompatible con el ideario nacionalista. cualquiera que sea el matiz político de éste.

Artículo $2^{2}$. Por los Talleres de nuestra dependencia se procederá a informarse si en sus cuadros existe algún miembro que actue activamente en el nacionalismo. En caso afirmativo se le hará ver la mencionada incompatibilidad. exhortándole se manifieste por cual de las dos situaciones opta y de preferir continuar en nuestra Augusta Institución se le pedirá declaración formal y escrita de tal resolución, especificando concretamente en la misma que deja en dicho momento de pertenecer a los miembros activos de la política nacionalista.

Artículo $3^{9}$-De existir algún miembro que no se someta a cuanto preceptúa el artículo anterior, será dado de baja en la Orden sin plancha de quite.

Artículo 4\%-Cópiese, cúmplase y dése cuenta a esta Gran Logia Regional. Dado en los Valles de Tetuán a 12 de julio de $1933 .{ }^{16}$

La reacción a dicho decreto fue bastante unánime, como consta en el expediente correspondiente del Archivo Histórico Nacional de Salamanca, donde, entre otros, se recoge el siguiente escrito dirigido a la Gran Logia Regional de Marruecos, desde Tetuán, el 15 de agosto de 1933:

"Después de dar lectura de la misma y del Decreto que en ella se adjunta os acusamos recibo de vuestra cariñosa plancha de 11 de julio $n^{2} 1.185$, cuyo decreto se refiere a la cuestión de los NACIONALISMOS. Os afirmamos con toda seguridad y certeza, que entre los miembros activos de este Respetable Taller no existe ningún hermano que simpatice con estos ideales NACIONALISTAS, que pugnan con los sanos elevados principios que patrocina nuestra Augusta Orden. De tal modo ha quedado eso acreditado en la observancia que todos y cada uno de nosotros hemos hecho de esos principios masónicos puros, incompatibles, como muy lógicamente decís, con el ideario NACIONALISTA, cualquiera que sea el matíz político de éste. ${ }^{17}$

Pocos días después, el 24 de agosto, era la logia Africa, de Tánger, la que sobre el mismo tema recibía la siguiente respuesta desde la sede de la Gran Logia Regional de Marruecos:

Contestamos a vuestra plancha 18 d/c en la que tratáis el asunto de NACIONALISMO. Nos alegra infinito que en ese taller no hay ningún hermano que sustente ideas NACIONALISTAS. El concepto que nosotros tenemos del Nacionalismo es el único que se puede tener y es, la aspiración a la hegemonía de una raza o de una idea sobre todas las demás; y como práctica política, la dictadura más antihumana y por lo tanto más antimasónica. Por las razones expuestas se expidió el DECRETO referente a este punto, en el que como habréis visto tienen cabida toda clase de nacionalismos, y sobre el que no cabe discusión

(16) Ibidem.

(17) Ibidem, Exp. 34. 
sino un estricto cumplimiento. ${ }^{18}$

En el fondo del problema, bastante más complejo de lo que a primera vista pudicra parecer, estaba el recuerdo todavía muy vivo de la situación que tuvo que afrontar el Grande Oriente Español, en agosto de 1896, cuando la polićáa de Madrid se incautó de los archivos masónicos así como de los de la asociación Hispano-Filipina, a lo que siguió el encarcelamiento y proceso de los responsables de ambas instituciones, acusados de haber fomentado el nacionalismo y posterior independencia de Filipinas, como luego lo serían de la de Cuba. Procesos que supusieron una crisis que duró cuatro años durante los cuales la vida masónica espańola estuvo prácticamente interrumpida, hasta que en 1900, a raíz del Congreso Masónico Internacional, celebrado en París del 31 de agosto al 2 de septiembre, del Grande Oriente Español recobró su actividad y reanudó la publicación de su Boletín Oficial -interrumpido desde septiembre de 1886- en cuyo número extraordinario del 10 de noviembre 1900 , se justificaban y felicitaban con estas palabras:

El Grande Oriente Español, que tanto trabajó masónica y profanamente en favor de la integridad de la Patria, y muy especialmente contra tendencia separatista y que cada día lamenta más la pérdida de nuestras antiguas posesiones de América y de Oceania, ve con gran satisfacción la fidelidad que a la masonería española guardan los masones de aquellas que fueron nuestras provincias, cuya mayoría continúa perteneciendo a nuestra federación, y engrandeciendo asi nuestra Orden.

Si contra nuestros propósitos y contra los suyos, hoy resultamos extranjeros, bueno es que sigamos siendo hermanos en Masoneria. ${ }^{19}$

(18) Ibidem. Estos escritos, con una lectura un tanto particular, están incluidos en un expediente que el fundador del entonces archivo policial de Salamanca, Marcelino de Ulibarri, califico de "Anti-Patria". Sobre el origen y vicisitudes de este archivo cfr. FERRER BENIMELI, José A., Archivo de la Guerra Civil, de Salamanca, Rev. Historia 16 (Madrid), $n^{9} 1.1311$, (enero 1982), págs. 109-115.

(19) Boletín Ofícial del Grande Oriente Español, $\mathrm{n}^{2}$ Extraordinario (16 noviembre 1900) pág. 4. 